\title{
Mechanistic Insights of Cells in Porous Scaffolds via Integrated Culture Technologies
}

\author{
Christopher Michael Gabbott and Tao Sun \\ Centre for Biological Engineering, Department of Chemical Engineering, Loughborough University, Loughborough, LE11 3TU, UK
}

\begin{abstract}
This research aimed to combine 3 cell and tissue culture technologies to obtain mechanistic insights of cells in porous scaffolds. When cultivated on 2D (2-dimensional) surfaces, HDFs (human dermal fibroblasts) behaved individually and had no strict requirement on seeding density for proliferation; while HaCat cells relied heavily on initial densities for proliferation and colony formation, which was facilitated when co-cultured with HDFs. Experiments using a 3D CCIS (3-dimensional cell culture and imaging system) indicated that HDFs colonised open pores of varying sizes (125-420 $\mu \mathrm{m})$ on modular substrates via bridge structures; while HaCat cells formed aperture structures and only colonised small pores (125 $\mu \mathrm{m})$. When co-cultured, HDFs not only facilitated HaCat attachment on the substrates, but also coordinated with HaCat cells to colonise open pores of varying sizes via bridge and aperture structures. Based on these observations, a 2-stage strategy for the culture of HDFs and HaCat cells on porous scaffolds was proposed and applied successfully on a cellulosic scaffold. This research demonstrated that cell colonisation in scaffolds was dependent on multiple factors; while the integrated 2D\&3D culture technologies and the 3D CCIS was an effective and efficient approach to obtain mechanistic insights of their influences on tissue regeneration.
\end{abstract}

Key words: Porous scaffold, cell colonisation, mechanistic understanding, 2D cell culture, 3D tissue culture, scale-down design.

\section{Introduction}

TE (Tissue Engineering) aims to apply the principles of engineering and life sciences toward the manufacturing of bio-substitutes to restore, maintain, enhance or replace failing human tissues or organs [1-3]. Since the first report of this research field over 30 years ago [4], dramatic advances and developments have been made. However, it is still a challenge task to manufacture fully functional tissues $[1,5,6]$. This is mainly due to several translational challenges. First of all, the mechanistic understanding of the relationships among molecules, cells, tissues and organs as a whole during tissue regeneration is somewhat limited [3, 7], even though the recognition and incorporation of these relationships and the underpinning biological processes is an inherent characteristic of $\operatorname{TE}[3,6]$. Secondly, there is no gold standard or generic procedure for tissue culture as a great amount of

Corresponding author: Tao Sun, Ph.D., senior lecturer, research field: tissue engineering. research efforts have been pursued to develop diverse 3D (three-dimensional) scaffolds, media and tissue culturing protocols [5, 7]. Thirdly, significant scale-up challenges still exist in TE [6], and new scale-up strategies based on thorough investigation of the infiltrated cells within the heterogeneous and anisotropic 3D scaffolds are urgently needed.

Insufficient understanding of tissue formation is the core of these translational challenges in TE. However, thorough investigation of the aforementioned relationships during tissue regeneration based on current cell and tissue culture technologies is still problematic. Notwithstanding its obvious advantages such as highly reproducible and cost-effective, the time-honored 2D (two-dimensional) cell culture is usually considered as a poor proxy of cells in vivo. This is because the 2D culture conditions are far removed from the complexities cells encounter in real-life tissues [8-10]. In vitro 3D tissue models thus have been developed to enhance the physiological relevance of experiments in vitro as they can provide 
more suitable 3D microenvironments for cell growth, differentiation and function [8-11]. However, the introduction of these in vitro models are a trade-off between the value of obtaining physiologically relevant data against their through-put as they are usually more labour intensive [11]. Furthermore, it is problematic to utilise tissue models for thorough investigation of the aforementioned complex relationships during tissue regeneration, as it is difficult to distinguish the regulatory functions of each individual architectural features at nano- and micro-scales, as well as other biochemical and biomechanical properties in the 3D culture environments [12-15]. To bridge the gap between the $2 \mathrm{D}$ and $3 \mathrm{D}$ culture technologies, we have recently developed a 3D CCIS (3D cell culture and imaging system) [15]. In this scale-down design, cells are cultivated on thin modular substrates with finely controlled open pores, and monitored non-invasively at single cell level using optical microscopes, thus it can be utilised as a high through-put platform to yield mechanistic insights of cell-cell and cell-scaffold interactions. As the 3D CCIS has a complete control of the structural features on the substrates as well as the biochemical and biomechanical properties within the 3D culture environments; their cellular regulatory functions can be distinguished and investigated separately.

This research aimed to combine current 2D\&3D culture technologies with the 3D CCIS in-between for the mechanistic understanding of HDFs (human dermal fibroblasts) and immortalised HaCat cells (human keratinocytes ) in a porous cellulosic scaffold. It was demonstrated that the integration of multiple culture technologies is an effective and efficient approach to obtain mechanistic insights of tissue formation, which can be utilised to inform the manufacture of fully functional tissues.

\section{Materials and Methods}

\subsection{Cell Culture}

Neonatal foreskin HDFs (Intercytex, Manchester
UK) and immortalised human keratinocytes (HaCaT cells, Addexbio, San Diego USA) were cultured in DMEM (Dulbecco's modified Eagle's medium, Lonza, Slough, UK) containing $4.5 \mathrm{~g} / \mathrm{L}$ glucose and supplemented with $10 \%$ (v/v) FBS (foetal bovine serum, Fisher Scientific, Loughborough, UK), $2 \mathrm{mM}$ L-glutamine (Sigma, Dorset, UK), 100 IU $/ \mathrm{mL}$ penicillin and $100 \mu \mathrm{g} / \mathrm{mL}$ streptomycin (Sigma, Dorset, UK), in cell culture T-flasks at $37{ }^{\circ} \mathrm{C}$ in $5 \%$ $\mathrm{CO}_{2}$ humidified atmosphere. Media in the flasks were changed twice a week and the cells were continually passaged prior to experimentation at $80-90 \%$ confluence using trypsin/EDTA (0.02\% (W/v) solution).

\subsection{Scaffolds and Modular Substrates for Cell Culture}

Due to its potential for medical applications, cellulose has been used for the fabrication of diverse porous scaffolds [16-18]. Therefore, a commercially available cellulosic scaffold (Azowipes ${ }^{\circledR}$ : non-woven viscose rayon bonded with a styrene buta-diene copolymer, Vernon-Carus Ltd., UK) was utilised in this study. The fibres of this scaffold were 20-50 $\mu \mathrm{m}$ in diameter, and the open pores were $50-300 \mu \mathrm{m}$ in diameter. The thickness of each scaffold layer was 0.1-0.2 $\mathrm{mm}$ and single layers were used for tissue culture. All the cellulosic scaffolds were thoroughly washed in PBS (Phosphate-buffered saline) and DMEM medium to remove all proprietary alcohol-based solvents in which they were soaked prior to cultivation of cells as previously described [19, 20], but variations were made in this study. Briefly, stainless steel ring (Outside diameter: $3.2 \mathrm{~cm}$; Inside diameter: $2.6 \mathrm{~cm}$ ) was placed on top of a single layer of cellulosic scaffold in 6-well plate, and $2.0 \mathrm{~mL}$ of cell culture medium was added into each well to submerge the scaffold. Different numbers of cells were then seeded inside the steel rings (Inside ring area: $5.31 \mathrm{~cm}^{2}$ ) to yield varying cell seeding densities. The cell scaffold composites were incubated in a tissue culture incubator at $37{ }^{\circ} \mathrm{C}$ and $5 \% \mathrm{CO}_{2}$ for 40 minutes to 18 hours, and then transferred into new 
wells of other 6-well plates with fresh media; which were replenished every three days. The cells that had filtrated through the porous scaffold and attached onto the original well surfaces were either disregarded or analysed via fluorescent microscopy for comparison purposes.

Commercial TEM (transmission electron microscopy) nickel specimen supporters (Diameter: $3.05 \mathrm{~mm}$, thickness: $10-30 \mu \mathrm{m}$, strut bar width: 40-80 $\mu \mathrm{m}$, Agar Scientific, Stansted, UK) with finely controlled square meshes of different sizes $(125,200$, 280 and $420 \mu \mathrm{m}$ ) were utilized as the modular substrates in the 3D CCIS. After washed thoroughly using distilled water, dried and autoclaved, the thin modular substrates were then suspended in the 3D CCISs for cell culture as previously described [15].

\subsection{Phase Contrast, Fluorescent and Scanning}

\section{Electron Microscopy}

The cells cultured on the surfaces of TCPs (tissue culture plates), cellulosic scaffolds, and suspended modular porous substrates were monitored and analysed noninvasively using an inverted Phase-Contrast Microscope (Nikon Eclipse Ti) during culture. The cells were also processed for SEM (scanning electron microscopy), or labelled with Cell Tracker $^{\mathrm{TM}}$ Red (CellTrackerTM CM-DiI Dye) or Green (CellTracker $^{\mathrm{TM}}$ Green CMFDA Dye) for live cell fluorescent microscopy as previously described [15]. To examine cell nuclei, the cells were fixed in IC (Intracellular) Fixation Buffer (Fisher Scientific) for 10 minutes, washed gently using PBS $(\times 3)$, stained with 7-AAD (7-Aminoactinomycin D, sigma) for 45 minutes, further washed using PBS $(\times 3)$ and then imaged at $\lambda_{\mathrm{ex}}=$ $546 \mathrm{~nm}, \lambda_{\text {em }}=647 \mathrm{~nm}$ (for TRITC/cell nuclei visualisation).

\subsection{Statistics}

Student's unpaired t-test was used to compare the seeded or cultivated cells in TCPs, cellulosic scaffolds, modular substrates or other culture conditions $\left({ }^{*} p<\right.$ $\left.0.05,{ }^{* *} p<0.01,{ }^{* * *} p<0.001\right)$.

\section{Results and Analysis}

\subsection{Mono- and Co-culture of HDFs and HaCat Cells} in $2 D$

Different numbers of HDFs and HaCaT cells (1000, 2000, 4000, 8000 cells $/ \mathrm{cm}^{2}$ for monocultures, or the same cell densities with 1:1 ratio of both cell types for co-cultures) were seeded in both 24-well tissue culture plates and T-flasks and cultured for 16 days. The cells were then monitored noninvasively via PCM (phase contrast microscope) on daily basis; some were also analysed using fluorescent microscopy and SEM. In monocultures almost all the HDFs were observed to migrate actively and behave individually until high confluences were obtained as illustrated in Figs. 1(AI-CI). The initial cell densities did not affect the survival of HDFs but demonstrated obvious influence on the time for the cells to reach full confluence. For example, when 8,000 cells $/ \mathrm{cm}^{2}$ were seeded, confluent HDFs were obtained within 2 days; while it took 7 days to reach $100 \%$ confluence if the initial density was 1,000 cells $/ \mathrm{cm}^{2}$ (Fig. 2A). In comparison, HaCat cells were less migratory and tended to aggregate with neighbouring cell(s) and form colonies for survival (Figs. 1(AII-CII)). Due to active cell proliferation and division, the HaCat colonies expanded outwards to accommodate new cells, and passive movement of HaCaT cells were observed as cell culture progressed. Initial HaCat density also demonstrated significant impact on the time to reach full confluence (Fig. 2B). If high seeding densities $\left(4,000\right.$ and 8,000 cells $\left./ \mathrm{cm}^{2}\right)$ were utilised, the majority of the HaCat cells could aggregate with neighbouring cell(s) to form relatively large colonies and confluent cultures were obtained within 7-9 days. If the initial density was reduced to 1,000 or 2,000 cells $/ \mathrm{cm}^{2}$, relatively small colonies were formed and the cells remained almost dormant for 2-3 days, then proliferated very slowly and only reached $10-20 \%$ confluence after 5-8 days' culture. 


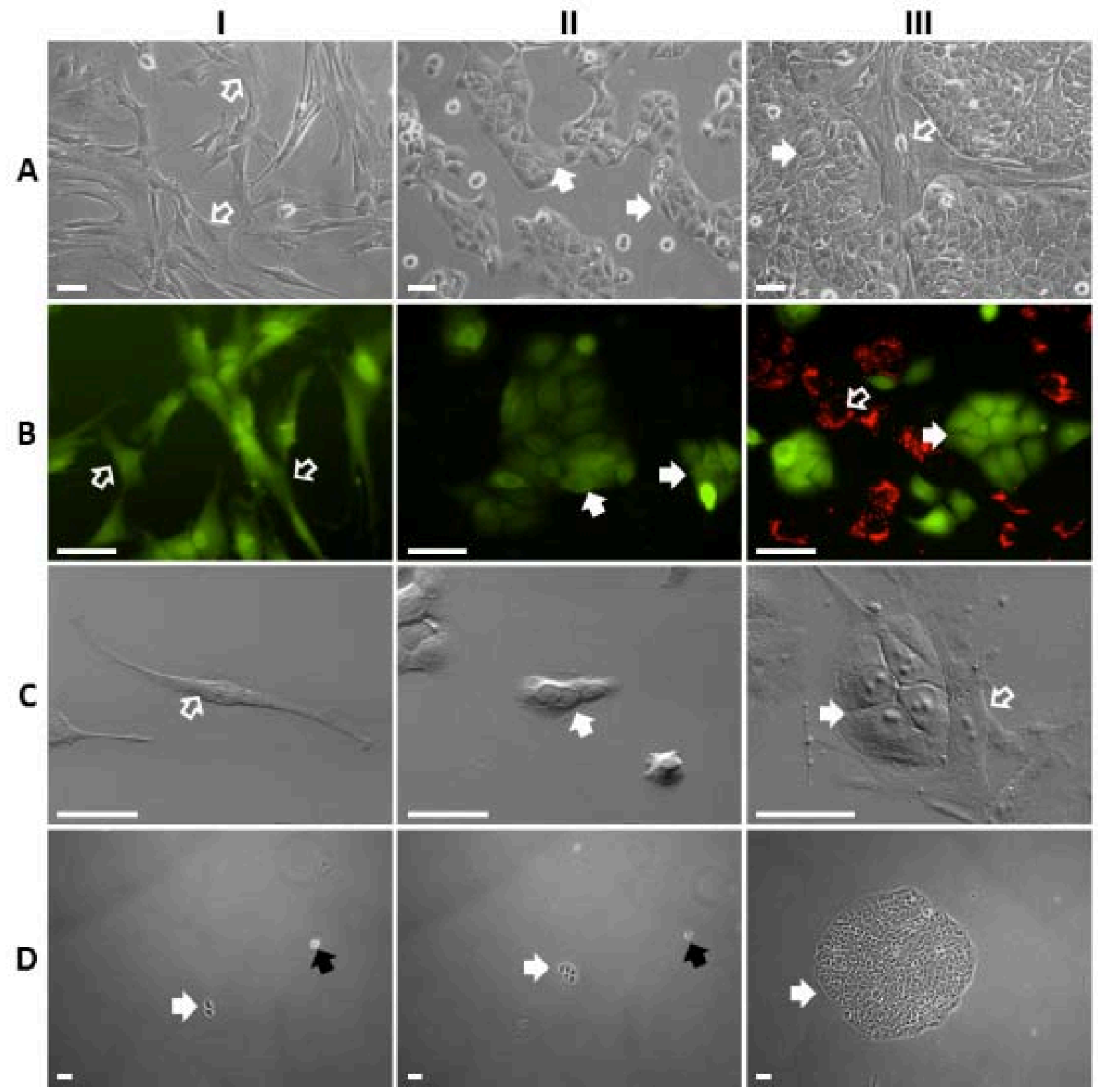

Fig. 1 Micrographs of Human dermal fibroblasts (HDFs, pointed by open arrows) and immortalised keratinocytes (HaCaT cells, pointed by close arrows) cultured on tissue culture surfaces. (A) Phase contrast, (B) fluorescent, (C) SEM micrographs of (I) HDFs, (II) HaCat cells in monocultures, (III) HDFs and HaCat cells in co-cultures. (D) Phase contrast micrographs of a HaCat colony with only 2 cells (pointed by white arrow) and a single HaCat cell (pointed by a black arrow) cultured for (I) 1 day, (II) 4 days, (III) 10 days. For fluorescent microscopy, HDFs and HaCat cells were stained using GREEN cell tracker in monocultures, while stained using RED and GREEN cell trackers respectively in co-cultures. Scale bar $=50 \mu \mathrm{m}$.

As more cells gradually accumulated within the colonies, they became even more proliferative and divided rapidly, and 90-100\% confluences were obtained after 16 days' culture. Further culture experiments using lower cell seeding density $\left(<100\right.$ cells $\left./ \mathrm{cm}^{2}\right)$ indicated that individual HaCat cells remained dormant without any obvious migration \& division, and gradually died out from the culture surfaces as shown in Figs. 1D(I-III). In very rare occasions, only 2-3 HaCat cells happened to form the smallest colonies probably because they were initially attached to each other due to the non-thorough trypsin treatment, or because they were seeded very closely on the tissue culture surface by coincidence. Microscopic analysis indicated that 
these 2-3 HaCat cells remained dormant initially and started to divide very slowly after 3-4 days' culture (Figs. 1D(I-II)). As more cells were created and the colony gradually expanded, the HaCat cells became even more proliferative, and a relatively large colony of approximately 400 cells could be obtained within 10 days of culture (Fig. 1DIII).

When co-cultured in 2D, the less migratory HaCaT cells aggregated to form colonies of varying sizes, which were surrounded by more motile individual HDFs (Figs. 1(AIII-CIII)). As the cultivation progressed, the surrounding HDFs would actively migrate away and gradually die out to accommodate the expanding $\mathrm{HaCaT}$ colonies, which eventually became the prominent cells. The initial HDF density had inverse influences on both the times for the cells to reach the highest confluences and the times for the
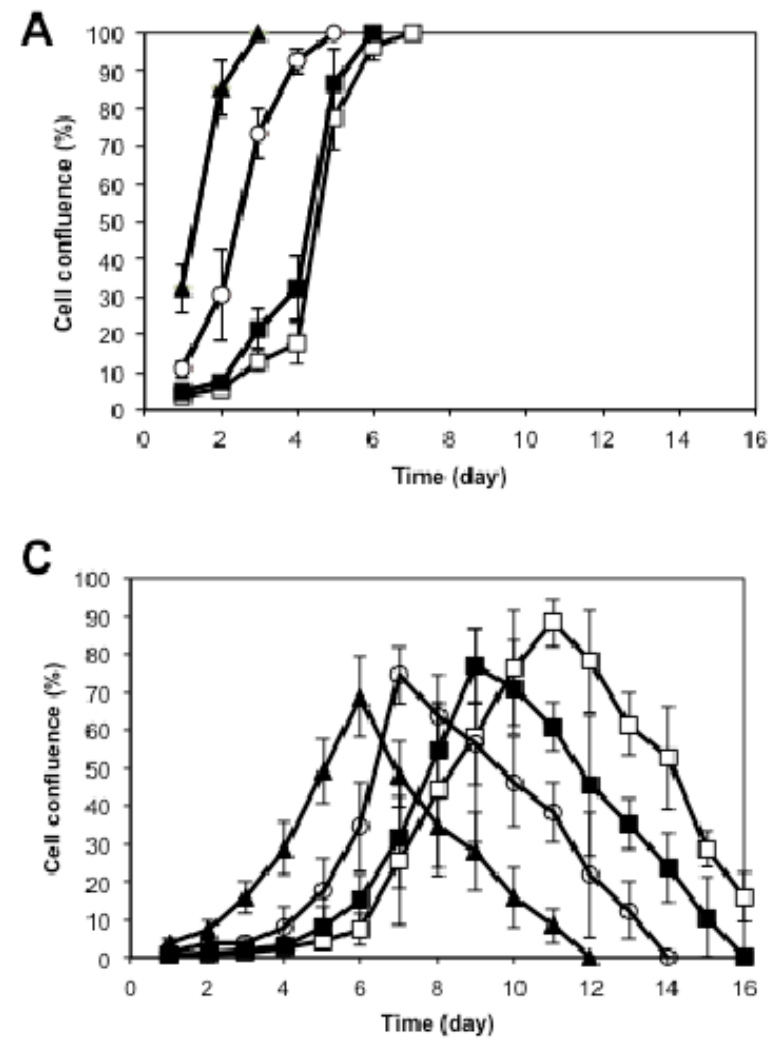

cells to die out (Fig. 2C); while the initial HaCat density demonstrated nearly linear impact on the times for the cells to reach full confluences as plotted in Fig. 2D.

\subsection{Mono- and Co-culture of HDFs and HaCat Cells} in the Ccellulosic Scaffolds

Preliminary experiments indicated that only a very small proportion of the seeded cells were able to attach onto the porous scaffolds as the majority of the cells filtrated through the scaffolds and eventually attached onto the tissue culture surfaces underneath. Therefore, the initial cell seeding densities within a very broad range $\left(2 \times 10^{2}, 2 \times 10^{3}, 2 \times 10^{4}, 2 \times 10^{5}, 2\right.$ $\times 10^{6}$ cells per $\mathrm{cm}^{2}$ of the cellulosic scaffold for monocultures, and the same cell densities with 1:1 ratio of both cell types for co-cultures) were tested on
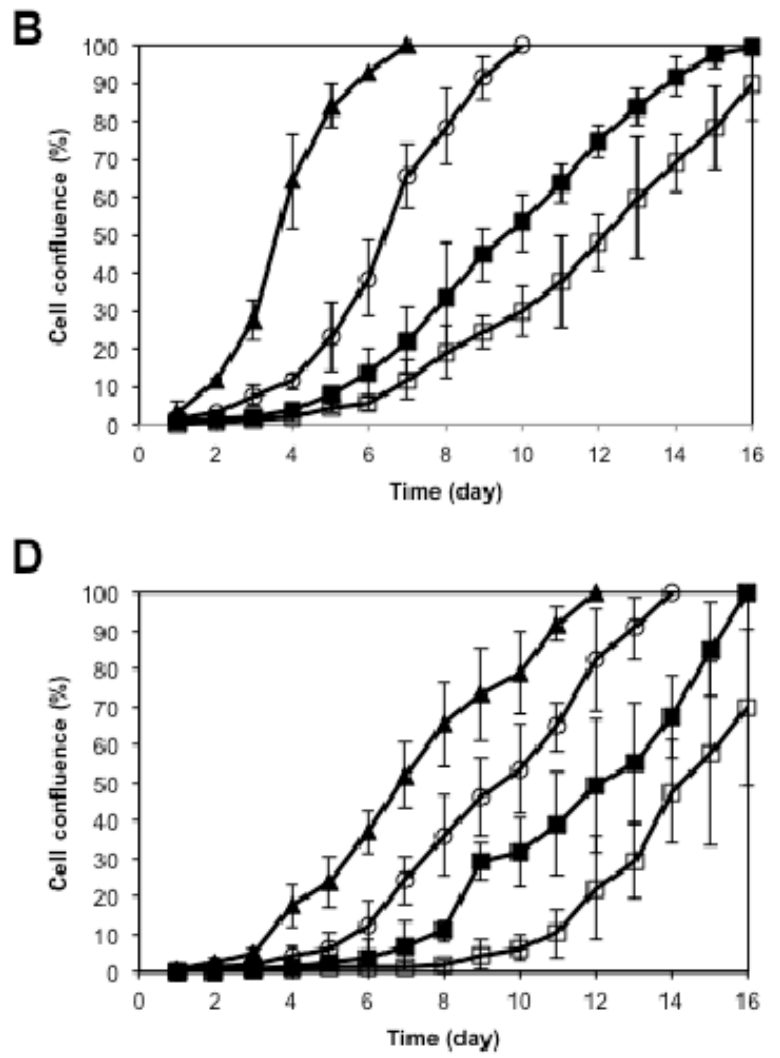

Fig. 2 The influences of cell seeding density and culture time on the confluence of cells cultured on tissue culture surfaces. Different numbers $\left(\mathbf{1 0 0 0}, \mathbf{2 0 0 0}, \circ 4000, \triangle 8000\right.$ cells $/ \mathrm{cm}^{2}$ ) of (A) Human dermal fibroblasts (HDFs), (B) immortalised keratinocytes (HaCaT) for monocultures; or the same cell densities with 1:1 ratio of (C) HDFs and (D) HaCat cells for co-cultures, were seeded on tissue culture surfaces and cultured for 16 days. Phase contrast micrographs were captured on daily basis and the cell confluences were analysed using Image J. Results shown are mean \pm SD. $(\mathbf{n}=3)$. 
this natural scaffold. The cells attached onto the scaffolds were continuously cultivated and carefully examined using different microscopic technologies. In monocultures some individual HDFs were observed to attach to the cellulosic fibres (Figs. 3(AI-BI)) or bridge the open pores (Fig. 3CI). However, the majority of the HDFs tended to work together and coordinate to form very complex cell networks within the open pores (Figs. 3(AII-CII)), which was significantly different from the 2D cultures. In comparison, HaCaT cells mono-cultured in the natural scaffolds aggregated to form cell spheres of varying sizes (Figs. 3(AIII-CIII), and Figs. 3(AIV-CIV)). Time-lapse experiments indicated that HDFs migrated actively either along the cellulosic fibres or along neighbouring cell(s) within the complex bridge structures; while the HaCat aggregates largely remained static within the scaffolds. As the elongated HDFs and the aggregated HaCat cells were still recognisable in co-cultures, some HDFs were observed to attach onto the HaCaT spheres
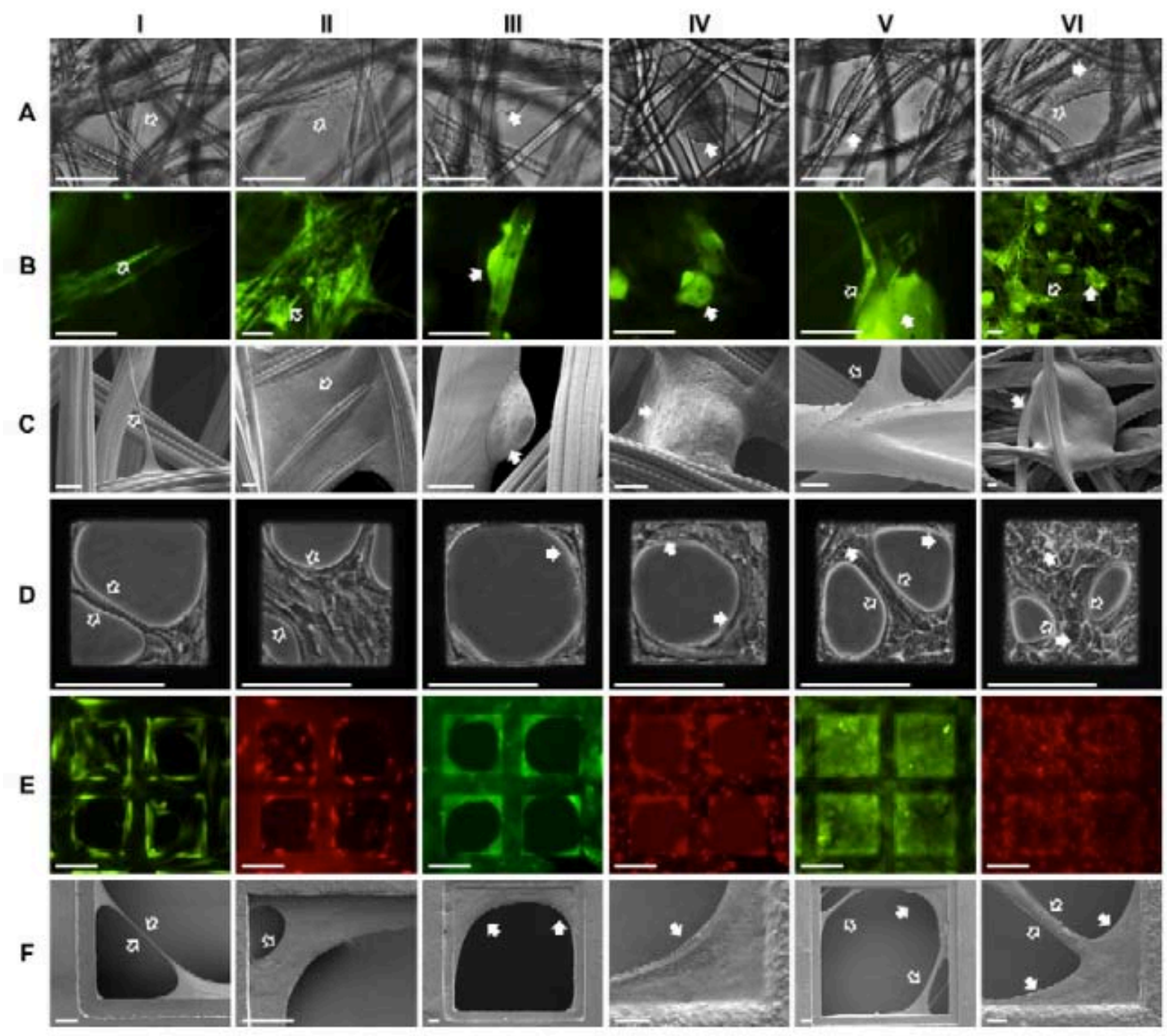

Fig. 3 Micrographs of Human dermal fibroblasts (HDFs, pointed by open arrows) and immortalised keratinocytes (HaCaT, pointed by close arrows) cultured in natural scaffolds and modular substrates. (A, D) Phase contrast, (B, E) fluorescent, (C, F) SEM (scanning electron microscopy) micrographs of (I-II) HDFs, (III-IV) HaCat cells, (V-VI) both HDFs and HaCat cells cultured on (A, B, C) cellulosic scaffolds, or (D, E, F) modular substrates with pore size of $125 \mu \mathrm{m}$. For fluorescent microscopy, HDFs and HaCat cells were stained using GREEN cell tracker, and nuclei stained with Red 7-AAD. Scale bars: $100 \mu \mathrm{m}$ (A, B, D, E), $10 \mu \mathrm{m}$ (C, F). 
and fix them within the porous scaffolds as shown in the PCM (Figs. 3A(V-VI)), fluorescent (Figs. $3 \mathrm{~B}(\mathrm{~V}-\mathrm{VI})$ ) and SEM micrographs (Figs. 3C(V-VI)), suggesting the strong interactions between these two cell types co-cultured in the natural scaffolds.

Due to the presence of randomly oriented fibres and open pores with irregular shapes and varying sizes, it was extremely difficult to accurately quantify cell colonisation within the cellulosic scaffolds using PCM, fluorescent microscopy and SEM as demonstrated in

A

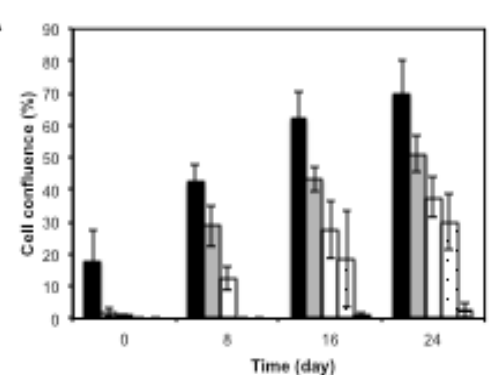

B

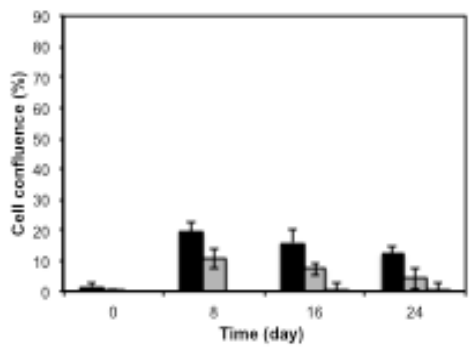

D
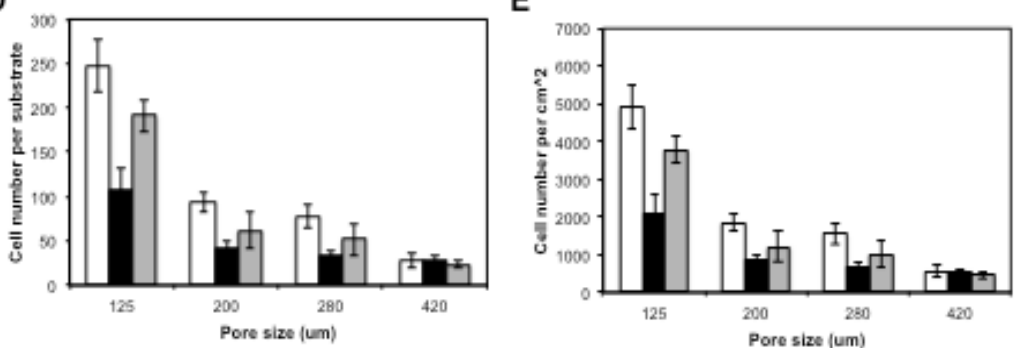

G

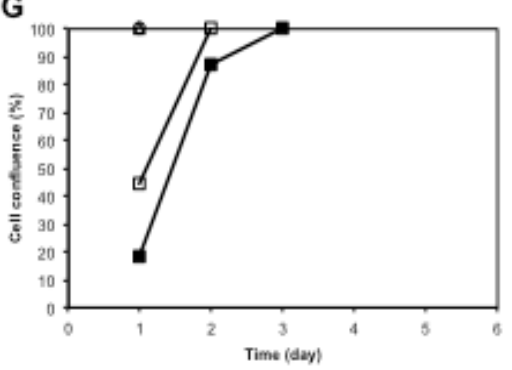

$\mathrm{H}$

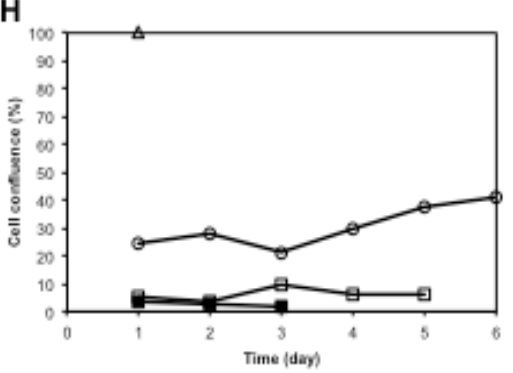

Figs. 3(A-C). Therefore, a fluorescent method was utilised to estimate the cell populations every 8 days for a period of 24 days. It was found that the initial cell density had nearly linear influences on the population of HDFs mono-cultured in the porous scaffolds, and the minimum seeding density to achieve detectable cell population ( $30 \%$ confluence) within 24 days was $2 \times 10^{3}$ cells $/ \mathrm{cm}^{2}$ (Fig. 4A). However, this linear relationship was not applicable for HaCat monocultures as illustrated in Fig. 4B. When large number of HaCat

C

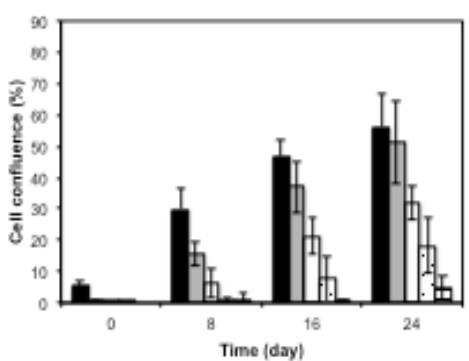

$\mathbf{F}$

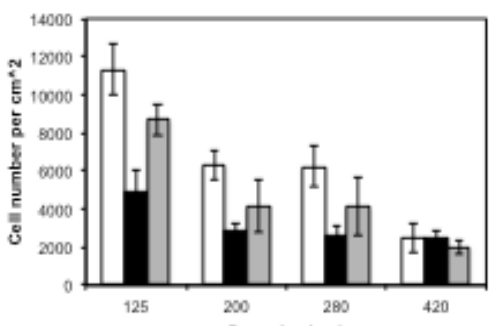

I

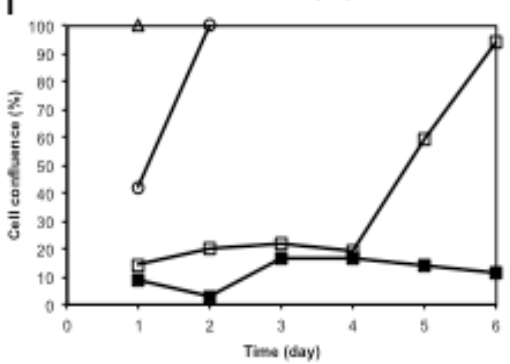

Fig. 4 The influences of cell seeding density and culture time period on cell confluence or attachment on cellulosic scaffolds or modular substrates. Different numbers (black: $2 \times 10^{6}$, grey: $2 \times 10^{5}$, white: $2 \times 10^{4}$, dotted: $2 \times 10^{3}$, dashed: $2 \times 10^{2}$ cells $/ \mathrm{cm}^{2}$ of cellulosic scaffolds) of (A) human dermal fibroblasts (HDFs), (B) immortalised keratinocytes (HaCaT) for monocultures, or $(\mathrm{C})$ the same cell densities with 1:1 ratio of both cell types for co-cultures, were seeded in the cellulosic scaffolds, stained using RED cell tracker and imaged at day 0, 8, 16 and 24 . Cell populations were approximated by analysing the percentages of the fluorescent areas in the images via Image $\mathrm{J}$. Aliquot of $200 \mathrm{uL}$ suspension $\left(1 \times 10^{5} \mathrm{cells} / \mathrm{ml}\right)$ of $\mathrm{HDFs}$ (white), or HaCaT cells (black) for monocultures, or the same cell densities with 1:1 ratio of HDFs and HaCat cells (grey) for co-cultures were seeded on the modular substrates with different pore sizes (125, 200, 280 and $420 \mu \mathrm{m})$, incubated for 40 minutes, the number of the cells (D) on each substrate, (E) per $\mathrm{cm}^{2}$ of substrate area, (F) per $\mathrm{cm}^{2}$ of strut area were calculated based on fluorescent images and plotted against the pore sizes. The confluences of (G) HDFs, (H) HaCat cells in monocultures; or (I) both cell types in co-cultures were analysed based on phase contrast micrographs and plotted against culture time periods. Results shown are mean \pm SD. $(n=3)$. 
cells were seeded $\left(2 \times 10^{5}\right.$ or $2 \times 10^{6}$ cells $\left./ \mathrm{cm}^{2}\right)$ into the porous scaffolds, only $10-20 \%$ cell confluences were detected at day 8 and 16, then the cell populations declined dramatically at day 24; and almost no detectable cells were obtained if less number of cells were seeded initially. In co-cultures the total cell densities were estimated, but it was not possible to distinguish the population of each cell type due to the limitations of the approximate fluorescent method. It was found that the cell seeding density had linear influence on the total cell populations; and the minimum initial density to achieve $\sim 30 \%$ confluence within 24 days was $2 \times 10^{4}$ cells $/ \mathrm{cm}^{2}$ (Fig. 4C), which was higher than that of HDF monocultures.

\subsection{Mono- and Co-culture of HDFs and HaCat Cells in $3 D$ CCIS}

Aliquot of $200 \mu \mathrm{L}$ HDF or HaCat suspension $(1 \times$ $10^{5}$ cells $/ \mathrm{mL}$ ) for monocultures, or the same cell densities with 1:1 ratio of both cell types for co-cultures were seeded onto each of the thin modular substrates with varying pore sizes (125, 200, 280 and $420 \mu \mathrm{m}$ ), which were then divided into 2 groups. In the 1st group, the cells were stained with red cell tracker prior to seeding, incubated for 40 minutes for their full attachment onto the modular substrates, and then imaged using fluorescent microscopy as shown in Fig. 5A. The total cell numbers on each substrate were registered and plotted against the pore sizes (Fig. 4D). It was found that the open pore size had inverse influence on cell attachment onto the substrates; as larger pore sizes were associated with less attached cells. Very similar cell numbers were detected on the substrates with the largest open pores $(420 \mu \mathrm{m})$ in mono- and co-cultures. On each of the substrates with small pores (125, 200 and $280 \mu \mathrm{m})$, significantly more HDFs were registered than HaCat cells in monocultures, while the total cell numbers in co-cultures were slightly higher than the numbers of HaCat cells, but less than the numbers of HDFs in monocultures. For the ease of comparison with 2D cell cultures, the numbers of the attached cells on per $\mathrm{cm}^{2}$ of modular substrate were then calculated and plotted against the open pore sizes as shown in Fig. 4E. Due to their strict requirements on initial seeding densities, only the HaCat cells mono-cultured on the modular substrate were carefully examined. It was found that the average HaCat density on the substrate with the smallest pore size $(125 \mu \mathrm{m})$ was approximately 2,000 cells per $\mathrm{cm}^{2}$ of substrate; while the densities on the other substrates (with the pore sizes of 200, 280 and $420 \mu \mathrm{m}$ ) were all less than 1,000 cells per $\mathrm{cm}^{2}$ of substrate (Fig. 4E). All these HaCat initial densities were only able to achieve low levels of cell populations (10-20\%) even if they were cultivated for 5-8 days in a very proliferative 2D culture environment as shown in Fig. 2B. The numbers of the attached cells on per $\mathrm{cm}^{2}$ of strut area of each modular substrate were also calculated (Fig. $4 \mathrm{~F})$. Since the open pores were excluded from the calculation, the HaCat density on the substrate with the smallest pore size $(125 \mu \mathrm{m})$ was 5,000 cells per $\mathrm{cm}^{2}$ of strut area; while the densities on the other substrates (with the pore sizes of 200, 280 and $420 \mu \mathrm{m}$ ) were all still less than 3,000 cells per $\mathrm{cm}^{2}$ of strut area (Fig. 4F).

To further investigate the influences of the initial densities on cell colonisation, the cells in the 2nd group were continuously cultured and analysed using different microscopic technologies. Live PCM analysis of the mono-cultures indicated that the majority of HDFs usually worked together and coordinated to form complex bridge structures across the open pores (Figs. 3D(I-II)), which were confirmed by fluorescent images (Figs. 3E(I-II)). Apart from these coordinated behaviors, highly elongated individual HDFs were also observed to attach both ends onto the struts to bridge small corners or voids as illustrated by the SEM images (Figs. 3F(I-II)). In comparison, HaCaT cells usually layered on top of each other to form aperture structures around the inner sides of the open pores, which then gradually 
expanded toward the central areas of the voids. Almost all the HaCat cells populated on the modular substrates were observed to form such colonies with aperture-shapes and very few behaved individually as illustrated in Figs. 3D (III-IV), Figs. 3E (III-IV) and Figs. 3F (III-IV). Systematic cultivation experiments demonstrated that these HaCat apertures were only able to completely fill small voids $(125 \mu \mathrm{m})$, but not any open pores larger than $200 \mu \mathrm{m}$. In co-cultures, both HDFs and HaCat cells maintained similar behaviours observed in monocultures. Moreover, HDFs were observed to form bridges within and or between HaCat apertures; meanwhile HaCat cells formed apertures along HDF bridges. Consequently, both cell types were able to utilise bridge and aperture structures to colonise within the open pores as demonstrated in Figs. 3D (V-VI) and Figs. 3E(V-VI). Apart from the coordinated behaviours in groups, highly elongated individual HDFs were also observed to attach both ends onto the substrate struts or HaCat apertures to bridge small corners or voids as illustrated in Figs. 3F(V-VI).

To accurately quantify cell colonization on the modular substrates, phase contrast micrographs of the cells within the open pores were captured on daily basis, based on which the highest confluences were analysed using Image $\mathrm{J}$ and registered. As shown in Fig. 4G, it only took HDFs 1 day to completely colonise small pores (125 and $200 \mu \mathrm{m}$ ), and 2-3 days to colonise bigger pores (280 and $420 \mu \mathrm{m}$ ). In comparison, HaCaT cells were able to completely cover the smallest void $(125 \mu \mathrm{m})$ within 1 day of culture, but only reached maximally $40 \%$ confluence in some of the slightly bigger pores $(200 \mu \mathrm{m})$ within 6 days' culture, and failed to fill the pores of 280 and $420 \mu \mathrm{m}$ in sizes as illustrated in Fig. 4H. In co-cultures, the voids of 125 and $200 \mu \mathrm{m}$ in sizes were completely filled by the cells with in 1 and 2 days respectively.
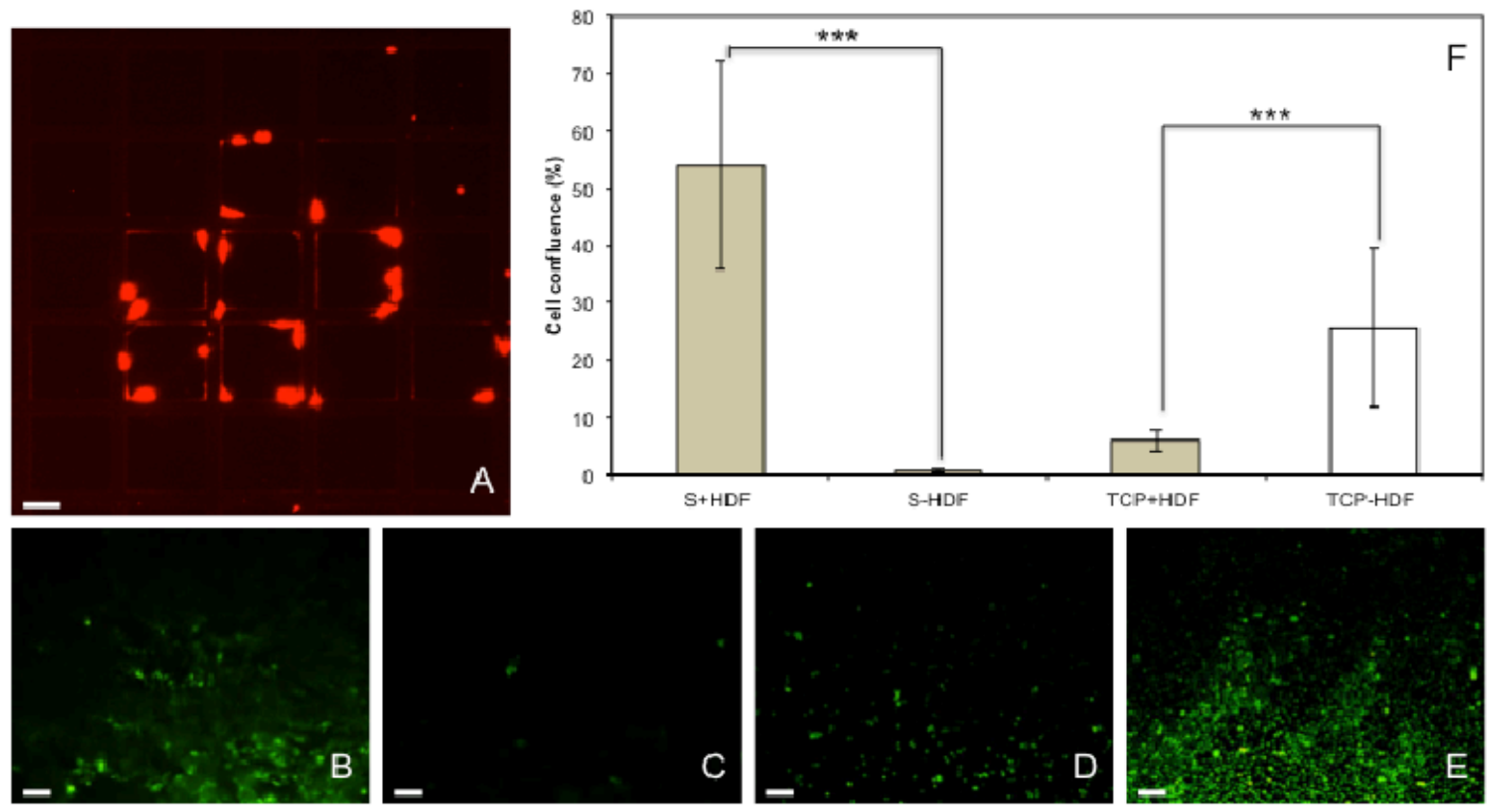

Fig. 5 (A) Fluorescent micrograph of Human dermal fibroblasts (HDFs) stained with RED cell tracker, seeded on the modular substrate with the pore size of $200 \mu \mathrm{m}$ and incubated for $\mathbf{4 0}$ minutes. (B-F) The influence of HDF on the attachment of HaCat cells on cellulosic scaffolds. HDFs $\left(2 \times 10^{5} \mathrm{cells} / \mathrm{cm}^{2}\right.$ of scaffold $)$ were seeded onto each piece of the cellulosic scaffold, cultured for 7 days; HaCat cells $\left(1 \times 10^{6} \mathrm{cells} / \mathrm{cm}^{2}\right)$ stained with GREEN cell tracker were then added onto each piece of cellulosic scaffold with or without the presence of HDFs and incubated for $\mathbf{4 0}$ minutes. Fluorescent micrographs of the HaCat cells (B) on the scaffolds with HDFs or (C) without HDFs; and (D) on the surfaces of tissue culture plastic underneath the scaffolds with HDFs or (E) without HDFs were captured. The fluorescent areas of each image was analysed with ImageJ to demonstrate $(F)$ the populations of HaCaT cells. Results shown are mean \pm SD. $(n=3)$. Scale bar $=100 \mu \mathrm{m}$. 

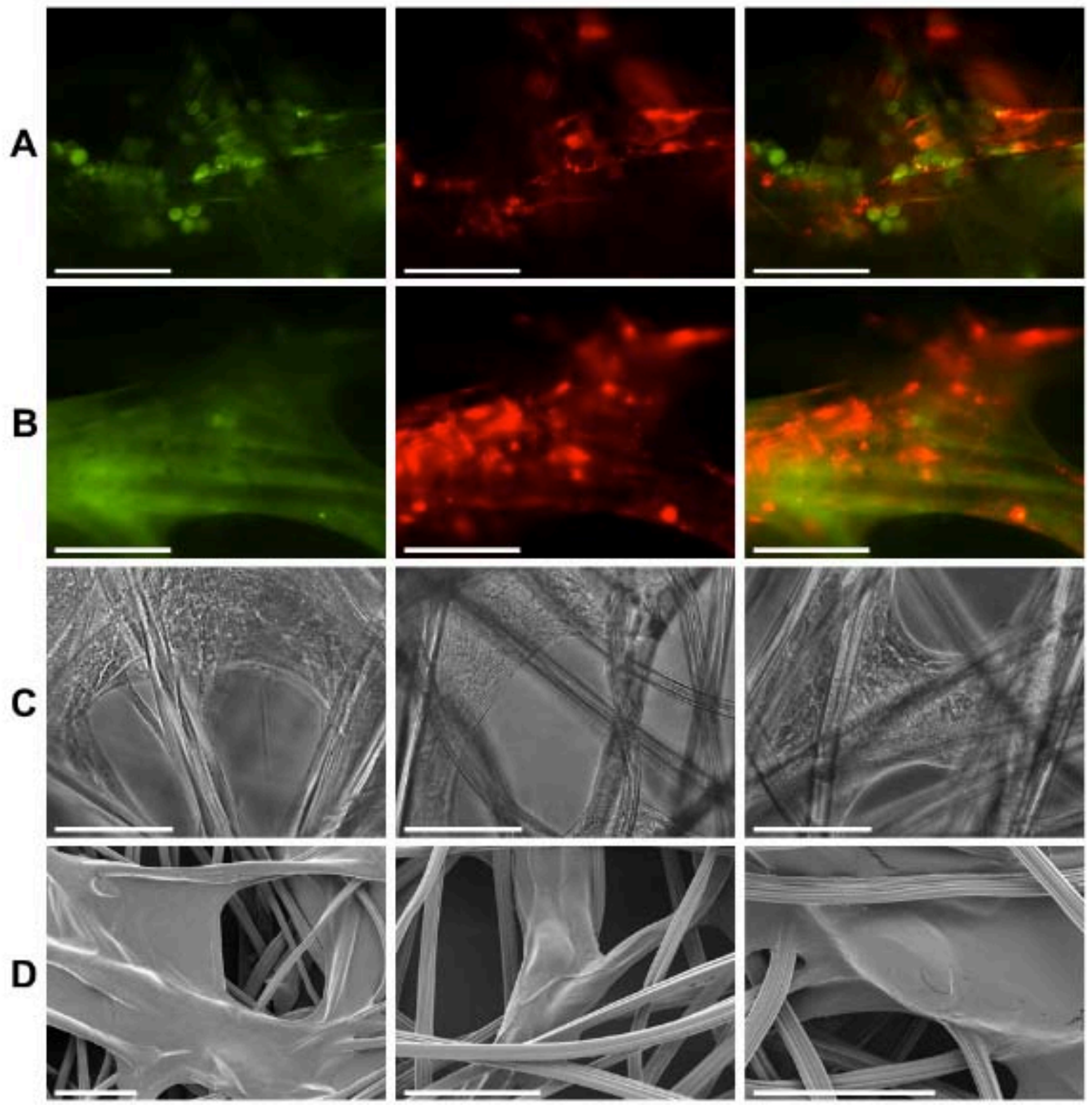

Fig. 6 Micrographs of Human dermal fibroblasts (HDFs) and immortalised keratinocytes (HaCaT) co-cultured in the cellulosic scaffolds using the 2-stage strategy. HDFs $\left(2 \times 10^{5} \mathrm{cells} / \mathrm{cm}^{2}\right.$ of scaffold $)$ stained with RED cell tracker were first seeded and cultured on each piece of cellulosic scaffolds for 7 days. HaCat cells $\left(1 \times 10^{6} \mathrm{cells} / \mathrm{cm}^{2}\right.$ of scaffold) stained with GREEN cell tracker were then seeded onto each of these scaffolds. Fluorescent micrographs of both cell types were captured (A) 1 hour, (B) 3 days after HaCat cell seeding. (C) Phase contrast and (D) scanning electron micrographs of the cells were also captured after 3 days' culture. Scale bar $=100 \mu \mathrm{m}$.

The cells managed to colonise the voids of $280 \mu \mathrm{m}$ within 6 days, but failed to cover the voids of $420 \mu \mathrm{m}$ (Fig. 4I).

\subsection{Two-Step Strategy to Culture HDFs and HaCaTs in the Cellulosic Scaffolds}

Based on the cultivation experiments in 2D and 3D
CCISs, it was hypothesised that certain level of HDF colonisation would facilitate HaCat attachment and proliferation in porous scaffolds. Therefore, a 2-step strategy was developed to co-culture HDFs and HaCat cells in the cellulosic scaffolds. HDFs $\left(2 \times 10^{5}\right.$ cells $/ \mathrm{cm}^{2}$ ) stained with red cell tracker were first seeded onto the natural scaffolds and cultured for 7 
days. HaCat cells $\left(1 \times 10^{6}\right.$ cells $\left./ \mathrm{cm}^{2}\right)$ stained with green cell tracker were then seeded onto the scaffolds with or without the presence of HDFs. After further incubated for 1 hour for full HaCat attachment, fluorescent micrographs of both cell types on the cellulosic scaffolds were captured as shown in Fig. 6A. In order to investigate the influence of colonised HDFs on HaCat attachment, the fluorescent micrographs of HaCat cells on the cellulosic scaffolds and the corresponding TCP surfaces underneath were captured (Figs. 5(B-E)) and analyzed using Image J. As shown in Fig. 5F, there were significantly more HaCat cells (55\% confluence) attached onto the porous scaffolds cultivated with HDFs than that without HDFs $(<3 \%)$. As expected, a large number of HaCat cells filtrated through the porous scaffolds without the presence of HDFs and eventually attached onto the corresponding TCP surfaces underneath in comparison with the TCPs underneath the scaffolds cultivated with HDFs (Fig. 5F).

After cultured for 3 days, the cell-scaffold composites were fixed for fluorescent microscopy and SEM analysis. It was found that HaCaT cells successfully colonised the scaffolds pre-cultured with HDFs. Further morphology analysis demonstrated that HaCaT cells attached onto the pre-colonised scaffolds and almost no cell aggregation was detected (Fig. 6B), which was confirmed by phase-contrast and high-quality SEM micrographs (Figs. 6(C-D)).

\section{Discussions}

In this research, 2D and 3D culture technologies were combined with a scale-down study design in-between to obtain mechanistic insights of cells in a porous scaffold.

Intensive 2D cell cultures were first carried out to investigate the main characteristics of different cell types. HDFs were observed to behave individually and have no strict requirement on seeding density for proliferation. Human keratinocytes usually form separate colonies within the epidermis and its appendages [21, 22], which has been explained as a genetically definable quantitative trait [23]. The keratinocyte colonies are subject to auto-regulation and can be recreated in 2D cell cultures [24, 25]. The immortalized HaCat cells still maintain the main characteristics of keratinocytes [26-29]. The capability of HaCat cells to colonise and proliferate was heavily depended on initial seeding densities. It was also significantly enhanced when co-cultured with HDFs. This was probably caused by the reported dynamic interactions between these 2 cell types, which encompassed soluble factors, extracellular matrix and direct cell-cell contacts [30-33].

Culture experimentations using the 3D CCIS demonstrated that HDFs could form complex bridge structures of single or multiple cell(s) and colonise within open pores of varying sizes $(125-420 \mu \mathrm{m})$, which was different from 2D cell cultures. In comparison, HaCat cells mainly grew on top of each other to form aperture structures within smaller pores (125 $\mu \mathrm{m})$ or corners, suggesting the formation of colonies as observed in 2D. The failure for HaCat cells to colonise on the substrates with large open pores can be explained as due to the unsatisfied minimum initial density for colony formation and cell survival as observed in 2D cultures. Co-cultures of HDFs and HaCat cells in the 3D CCIS indicated that the presence of HDFs on the substrates facilitated the attachment and colony formation of HaCat cells, which echoed the observations from 2D cultures. Moreover, HaCat cells were observed to coordinate with HDFs to colonise open pores of varying sizes (125-280 $\mu \mathrm{m})$ using both bridge and aperture structures. Based on the thorough investigations of cell-ell and cell-substrate interactions using both 2D cell culture and the scale-down design, a 2-stage strategy for the culture of HDFs and HaCat cells was then designed and successfully applied for tissue culture using the cellulosic scaffold.

This research demonstrated that even though it is a poor proxy, the time-honoured 2D cell culture is still a 
convenient and valuable technology for the understanding of the basic cell properties such as HaCat colony formation, which can be utilised for the design and understanding of 3D tissue cultures using either natural scaffolds or modular substrates. Tissue culture using real scaffold(s) is a good method to identify various factors such as open pore size, cell type, cell-cell interaction and initial cell density that influence cell colonization, but it is difficult to accurately investigate the action mechanisms of these variables. Based on the main cell characteristics observed through 2D cell cultures and the factors identified via 3D tissue cultures, specific experiments could be designed and performed on the 3D CCIS to not only accurately analyse cell-substrate and cell-cell interactions at single-cell level, but also design and evaluate new cell seeding and culturing methodologies for tissue cultures on complex scaffold(s). Therefore, all these 3 technologies can be closely integrated as an effective approach to obtain the mechanistic insights of tissue formation, and to inform the manufacturing of fully functional tissues for clinical and diagnostic purposes.

\section{Acknowledgements}

This study was funded by the Engineering and Physical Sciences Research Council (EPSRC; UK) (EP/L015072/1).

\section{References}

[1] Alberti, C. 2009. “Tissue Engineering Technologies: Just a Quick Note about Transplantation of Bioengineered Donor Trachea and Augmentation Cystoplasty by De Novo Engineered Bladder Tissue.” G Chir. 30: 514-9.

[2] Zonari, A., Novikoff, S., Electo, N. R. P., Breyner, N. M., Gomes, D. A., and Martins, A. 2012. "Endothelial Differentiation of Human Stem Cells Seeded onto Electrospun

Polyhydroxybutyrate/Polyhydroxybutyrate-Co-Hydroxyv alerate Fiber Mesh.” PLoS ONE 7 (4): e35422.

[3] Gurtner, G. C., and Chapman, M. A. 2016. "Regenerative Medicine: Charting a New Course in Wound Healing.” Adv Wound Care 5: 314-28.

[4] Langer, R., and Vacanti, J. P. 1993. "Tissue
Engineering.” Science 260: 920-6.

[5] Baiguera, S., Urbani, L., and Del Gaudio, C. 2014. "Tissue Engineered Scaffolds for an Effective Healing and Regeneration: Reviewing Orthotopic Studies." Biomed Res Int. 2014: e398069.

[6] Scarritt, M. E., Pashos, N. C., and Bunnell, B. 2015. “A Review of Cellularization Strategies for Tissue Engineering of Whole Organs.” Front Bioeng Biotechnol. 3: 1-17.

[7] de Kemp, V., de Graaf, P., Fledderus, J. O., Ruud Bosch, J. L. H., and de Kort, L. M. O. 2015. “Tissue Engineering for Human Urethral Reconstruction: Systematic Review of Recent Literature.” PLoS ONE. 10: e0118653.

[8] de la Puente, P., Muz, B., Gilson, R. C., Azab, F., Luderer, M., King, J., Achilefu, S., Vij, R., and Azab, A. K. 2015. "3D Tissue-Engineered Bone Marrow as a Novel Model to Study Pathophysiology and Drug Resistance in Multiple Myeloma.” Biomaterials 73: 70-8.

[9] Knight, E., and Przyborski, S. 2015. “Advances in 3D Cell Culture Technologies Enabling Tissue-Like Structures to Be Created in Vitro.” J Anat. 227: 746-56.

[10] Rogozhnikov, D., O'Brien, P. J., Elahipanah, S., and Yousaf, M. N. 2016. "Scaffold Free Bio-orthogonal Assembly of 3-Dimensional Cardiac Tissue via Cell Surface Engineering.” Sci Rep. 6: e39806.

[11] Wrzesinski, K., and Fey, S. J. 2015. "From 2D to 3D-A New Dimension for Modelling the Effect of Natural Products on Human Tissue." Curr Pharm Des. 21: 5605-16.

[12] Lin, H., Godiwalla, S. Y., Palmer, B., Frimberger, D., Yang, Q., Madihally, S. V., Fung, K., and Kropp, B. P. 2014. "Understanding Roles of Porcine Small Intestinal Submucosa in Urinary Bladder Regeneration: Identification of Variable Regenerative Characteristics of Small Intestinal Submucosa.” Tissue Eng Part B Rev. 20: 73-83.

[13] Yang, C., Deng, G., Chen, W., Ye, X., and Mo, X. 2014. "A Novel Electrospun-Aligned Nanoyarn-Reinforced Nanofibrous Scaffold for Tendon Tissue Engineering." Colloids Surf B. 122: 270-6.

[14] Lowenthal, J., and Gerecht, S. 2016. "Stem Cell-Derived Vasculature: A Potent and Multidim Ensional Technology for Basic Research, Disease Modeling, and Tissue Engineering." Justin Biochem Biophys Res Commun 473: 733-42.

[15] Gabbott, C. M., Zhou, Z. X., Han, G. X., and Sun, T. 2017. “A Novel Scale-down Cell Culture and Imaging Design for the Mechanistic Insight of Cell Colonization within Porous Substrate.” J. Microsc. 267: 150-6.

[16] Ramli, N. A., and Wong, T. W. 2011. "Sodium Carboxymethylcellulose Scaffolds and Their Physicochemical Effects on Partial Thickness Wound 
Healing.” Int. J. Pharm. 403: 73-82.

[17] Jonsson, M., Brackmann, C., Puchades, M., Brattås, K., Ewing, A., Gatenholm, P., and Enejder, A. 2015. "Neuronal Networks on Nanocellulose Scaffolds.” Tissue Eng. Part C Methods 21: 1162-70.

[18] Abraham, E., Weber, D. E., Sharon, S., Lapidot, S., and Shoseyov, O. 2017. "Multifunctional Cellulosic Scaffolds from Modified Cellulose Nanocrystals.” ACS Appl Mater Interfaces 9: 2010-5.

[19] Sun, T., Norton, D., Haycock, J. W., Ryan, A. J., and MacNeil, S. 2005. "Development of a Closed Bioreactor System for Culture of Tissue-Engineered Skin at an Air-Liquid Interface.” Tissue Eng. 11: 1824-31.

[20] Sun, T., Jackson, S., Haycock, J. W., and MacNeil, S. 2006. "Culture of Skin Cells in 3D Rather than 2D Improves Their Ability to Survive Exposure to Cytotoxic Agents.” J. Biotechnol. 122: 372-81.

[21] Kobayashi, K., Rochat, A., and Barrandon, Y. 1993. "Segregation of Keratinocyte Colony-Forming Cells in the Bulge of the Rat Vibrissae.” PNAS 90: 7391-5.

[22] Wiener, D. J., Doherr, M. G., Müller, E. J., and Welle, M. M. 2016. "Spatial Distribution of Stem Cell-Like Keratinocytes in Dissected Compound Hair Follicles of the Dog.” PLoS One 11: e0146937.

[23] Popova, N. V., Tryson, K. A., Wu, K. Q., and Morris, R. J. 2002. "Evidence that the Keratinocyte Colony Number is Genetically Controlled.” Exp. Dermatol. 11: 503-8.

[24] Jensen, U. B., Lowell, S., and Watt, F. M. 1999. “The Spatial Relationship between Stem Cells and their Progeny in the Basal Layer of Human Epidermis: a New View Based on Whole-Mount Labelling and Lineage Analysis.” Development 126: 2409-18.

[25] Savill, N. J., and Sherratt, J. A. 2003. "Control of Epidermal Stem Cell Clusters by Notch-Mediated Lateral Induction.” Dev Biol. 258: 141-53.
[26] George, V. C., Naveen Kumar, D. R., Suresh, P. K., Kumar, S., and Kumar, R. A. 2013. "Comparative Studies to Evaluate Relative in Vitro Potency of Luteolin in Inducing Cell Cycle Arrest and Apoptosis in HaCaT and A375 cells.” Asian Pac J Cancer Prev. 14: 631-7.

[27] Bastianetto, S., Dumont, Y., Duranton, Y., Vercauteren, F., Breton, L., and Quirion, R. 2010. "Protective Action of Resveratrol in Human Skin: Possible Involvement of Specific Receptor Binding Sites.” PLoS One 5: e12935.

[28] Mantso, T., Sfakianos, A. P., Atkinson, A., Anestopoulos, I., Mitsiogianni, M., Botaitis, S., Perente, S., Simopoulos, C., Vasileiadis, S., Franco, R., Pappa, A., and Panayiotidis, M. I. 2016. "Development of a Novel Experimental in Vitro Model of Isothiocyanate-Induced Apoptosis in Human Malignant Melanoma Cells." Anticancer Res. 36: 6303-9.

[29] Leung, K. S., Chan, H. F., Leung, H. H., Galano, J. M., Oger, C., Durand, T., and Lee, J. C. 2017. "Short-Time UVA Exposure to Human Keratinocytes Instigated Polyunsaturated Fatty Acid without Inducing Lipid Peroxidation.” Free Radic Res. 51: 269-80.

[30] Gailit, J., and Clark, R. A. F. 1994. "Wound Repair in the Context of Extracellular Matrix.” Curr Opin Cell Biol. 6: 717-25.

[31] Maas-Szabowski, N., Shimotoyodome, A., and Fusenig, N. E. 1999. "Keratinocyte Growth Regulation in Fibroblast Co-cultures via a Double Paracrine Mechanism.” J. Cell Sci. 112: 1843-53.

[32] Szabowski, A., Maas-Szabowski, N., Andrecht, S., Kolbus, A., Kistner, M. S., Fusenig, N. E., and Angel, P. 2000. "C-Jun and Jun-B Antagonistically Control Cytokine-Regulated Mesenchymal-Epidermal Interaction in Skin.” Cell 103: 745-55.

[33] Angel, P., and Szabowski, A. 2002. "Function of AP-1 Target Genes in Mesenchymal-Epithelial Cross-Talk in Skin.” Biochem. Pharmacol. 64: 949-56. 